\title{
$\beta$-Lactamase production and antibiotic susceptibility pattern of Moraxella catarrhalis isolates collected from two county hospitals in China
}

Wei Shi ${ }^{1+}$, Denian Wen ${ }^{2+}$, Changhui Chen ${ }^{3+}$, Lin Yuan ${ }^{1}$, Wei Gao ${ }^{1}$, Ping Tang ${ }^{2}$, Xiaoping Cheng $^{3}$ and Kaihu Yao ${ }^{{ }^{*}}$ (I)

\begin{abstract}
Background: Moraxella catarrhalis (M. catarrhalis) is an important bacterial pathogen. However, its antibiotic susceptibility patterns in different areas are difficult to compare because of the use of different methods and judgement criteria. This study aimed to determine antimicrobial susceptibility and $\beta$-lactamase activity characteristics of $M$. catarrhalis isolates collected from two county hospitals in China, and to express the results with reference to three commonly used judgement criteria.
\end{abstract}

Results: Nasopharyngeal swabs were obtained from child inpatients with respiratory tract infections at the People's Hospital of Zhongjiang County and Youyang County from January to December 2015. M. catarrhalis strains were isolated and identified from the swabs, and susceptibility against 11 antimicrobials was determined using the E-test method or disc diffusion. Test results were interpreted with reference to the standards of the European Committee on Antimicrobial Susceptibility Testing (EUCAST), the Clinical and Laboratory Standards Institute (CLSI), and the British Society for Antimicrobial Chemotherapy (BSAC). Detection of $\beta$-lactamase activity was determined by the chromogenic cephalosporin nitrocefin. M. catarrhalis yield rates were 7.12 and 9.58\% (Zhongjiang County, 77/1082 cases; Youyang County, 101/1054 cases, respectively). All isolates were susceptible to amoxicillin-clavulanic acid. The susceptibility rate to meropenem was 100\% according to EUCAST; no breakpoints were listed in CLSI or BSAC. The non-susceptibility rate to sulfamethoxazole-trimethoprim differed significantly between the two hospitals regardless of the judgemnet criteria used, with isolates from Zhongjiang showing higher susceptibility to those from Youyang (Fisher's exact test, $P<0.05$ ). According to CLSI, the total non-susceptibility rate to erythromycin was 70.8\% (Zhongjiang County, 79.2\%; Youyang County, 64.3\%), and the rate reached 92.1\% (Zhongjiang County, 90.9\%; Youyang County, 93.1\%) on the basis of EUCAST or BSAC. The total positive rate of $\beta$-lactamase was $99.4 \%$ (177/178 cases) (Zhongjiang County, 100\%, 77/77 cases; Youyang County, 99.0\%, 100/101 cases).

Conclusions: Ninety nine percent of $M$. catarrhalis isolates produce $\beta$-lactamase. The isolates showed poor susceptibility to ampicillin and erythromycin, and high susceptibility to the third- and fourth-generation cephalosporins and amoxicillin-clavulanic. Significant discrepancies between different antimicrobial susceptibility judgemnet criteria were noted.

Keywords: Moraxella catarrhalis, Antibiotic resistance, $\beta$-Lactamase, Children

\footnotetext{
*Correspondence: Jiuhu2655@sina.com

${ }^{+}$Wei Shi, Denian Wen and Changhui Chen contributed equally to this work.

${ }^{1}$ Key Laboratory of Major Diseases in Children, National Key Discipline of

Pediatrics (Capital Medical University), National Clinical Research Center for

Respiratory Diseases, Beijing Key Laboratory of Pediatric Respiratory Infection

Diseases, Beijing Pediatric Research Institute, Beijing Children's Hospital,

Capital Medical University, No. 56 Nan-li-shi Road, Beijing 100045, China

Full list of author information is available at the end of the article
}

(c) The Author(s). 2018 Open Access This article is distributed under the terms of the Creative Commons Attribution 4.0 International License (http://creativecommons.org/licenses/by/4.0/), which permits unrestricted use, distribution, and reproduction in any medium, provided you give appropriate credit to the original author(s) and the source, provide a link to the Creative Commons license, and indicate if changes were made. The Creative Commons Public Domain Dedication waiver (http://creativecommons.org/publicdomain/zero/1.0/) applies to the data made available in this article, unless otherwise stated. 


\section{Background}

Moraxella catarrhalis ( $M$. catarrhalis), also known as Micrococcus catarrhalis, Neisseria catarrhalis, and Branhamella catarrhalis is a gram-negative, aerobic, oxidase-positive diplococcus. It is a commensal species that is exclusively a human pathogen of the upper respiratory tract. However, it is now recognized as causative of otitis media in children and acute exacerbations of chronic obstructive pulmonary disease in adults $[1,2]$. M. catarrhalis can also cause endocarditis, sepsis and meningitis, though these occur more rarely [1].

Community-acquired pneumonia (CAP) is a major cause of morbidity and mortality in children worldwide, and $M$. catarrhalis is the third most common causative pathogenic bacteria of CAP, following Streptococcus pneumoniae and Haemophilus influenzae [3]. The importance of $M$. catarrhalis in CAP infection is also increasing with the introduction of the pneumococcal conjugate vaccine and Haemophilus influenzae type b vaccine.

The first $\beta$-lactamase positive M. catarrhalis isolate was reported in 1977 in Sweden [4]. Since then, $\beta$-lactamase production has been reported from various countries with different frequencies, which mostly exceed 90.0\% [3, 5-7], revealing the poor antibiotic susceptibility pattern of the isolate. Three antimicrobial resistance judgemnet criteria can be used to analyze the antibiotic susceptibility pattern of $M$. catarrhalis strains: the European Committee on Antimicrobial Susceptibility Testing (EUCAST), the Clinical and Laboratory Standards Institute (CLSI) [8], and the British Society for Antimicrobial Chemotherapy (BSAC) [9]; CLSI is most commonly used in China. Because of the lack of a unified testing method and judgemnet criteria for antibiotic resistance of $M$. catarrhalis, data from different areas show significant differences [10, 11]. Moreover, susceptibility testing of $M$. catarrhalis is not routinely performed in most diagnostic laboratories, and many resistance data derive from large cities, with few studies conducted in more rural areas. Therefore, the aim of this study was to determine the level of $\beta$-lactamase production and antibiotic resistance patterns of $M$. catarrhalis isolates collected from two county hospitals in China.

\section{Methods}

\section{Study population}

Nasopharyngeal swabs were collected from child inpatients with respiratory tract infections at the People's Hospitals of Zhongjiang County and Youyang County, located in western China, from January to December 2015. Children meeting the following criteria were included: (1) aged between 1 month and 14 years; (2) having an infection for fewer than 3 days; (3) having a fever and cough; and (4) with permission from a parent and/or legal guardian. Children with factors potentially affecting the acquisition of nasopharyngeal swab specimens, such as repeated tic, and coagulation disorders, were excluded.

A total of 1082 children were included from Zhongjiang County, of whom 642 were male and 440 were female; a total of 1054 were included from Youyang County, of whom 679 were male and 375 were female. Chocolate agar and blood agar were used to culture the specimens, which were incubated for $24 \mathrm{~h}$ at $37{ }^{\circ} \mathrm{C}$. Bacterial growth was identified by the examination of colony morphology, gram staining, and microscopic analysis. Additional identification tests were the oxidase test, catalase test, carbohydrate fermentation test, DNAse test, and nitrate reduction test.

A parent and/or legal guardian of each participant signed a written informed consent document before enrollment and before any study procedure was performed. This study was approved by the Ethics Committee of the two hospitals (Ethics Committee of People's Hospital of Zhongjiang County, and Ethics Committee of People's Hospital of Chongqing Youyang County). No ethical problems were encountered in this study.

\section{Antimicrobial susceptibility and $\beta$-lactamase testing}

The minimum inhibitory concentrations (MICs) of all isolates were determined against ampicillin, amoxicillinclavulanic acid, cefuroxime, ceftazidime, cefepime, ciprofloxacin, erythromycin, and meropenem using E-test strips (AB Biodisk, Solna, Sweden), and their susceptibility to sulfamethoxazole-trimethoprim, chloramphenicol, and tetracycline was assessed with disc diffusion tests (Oxoid, Basingstoke, England). The results were interpreted with reference to the standards of EUCAST(http://www.eucast.org/ clinical_breakpoints/), CLSI [8], and BSAC [9], and breakpoints are listed in Table 1. $\beta$-lactamase activity was determined by the chromogenic cephalosporin nitrocefin (BR66A; Oxoid). Streptococcus pneumoniae ATCC49619 was used as the quality control strain in susceptibility tests.

\section{Statistical analysis}

Antimicrobial resistance data were analyzed with WHONET 5.6 software as recommended by the World Health Organization. Statistical analyses were performed using SPSS software 17.0 (SPSS, Chicago, IL). The $x^{2}$ test and Fisher's exact test were used for significant comparisons between groups. $P$ values $<0.05$ were deemed to indicate statistical significance.

\section{Results}

A total of 77 and $101 \mathrm{M}$. catarrhalis isolates were separately detected from Zhongjiang and Youyang counties, respectively, with yield rates of $7.12 \%$ (77/1082 cases) and $9.58 \%$ (101/1054 cases). There was no significant difference in the yield rate between boys $(6.7 \% ; 43 / 642$ 
Table 1 Antibiotic breakpoints for M. catarrhalis strains in EUCAST, CLSI and BSAC

\begin{tabular}{|c|c|c|c|c|c|c|c|c|c|}
\hline \multirow[t]{2}{*}{ Antibiotics $^{*}$} & \multirow[t]{2}{*}{$\mathrm{MIC}^{\mathrm{a}} / \mathrm{D}$-zone } & \multicolumn{2}{|c|}{ EUCAST $^{c}$} & \multicolumn{3}{|l|}{$\mathrm{CLSI}^{\mathrm{d}}$} & \multicolumn{3}{|l|}{$\mathrm{BSAC}^{\mathrm{e}}$} \\
\hline & & $S$ & $\mathrm{R}$ & $S$ & I & $\mathrm{R}$ & $S$ & I & $\mathrm{R}$ \\
\hline AMP & $\mathrm{MIC}(\mathrm{mg} / \mathrm{L})$ & -\# $^{-1}$ & -\# & $-\#$ & $-\#$ & $-\#$ & $\leq 1$ & - & $>1$ \\
\hline AMC & $\mathrm{MIC}(\mathrm{mg} / \mathrm{L})$ & $\leq 1$ & $>1$ & $\leq 4$ & - & $\geq 8$ & $\leq 1$ & - & $>1$ \\
\hline CXM & $\mathrm{MIC}(\mathrm{mg} / \mathrm{L})$ & $\leq 4$ & $>8$ & $\leq 4$ & 8 & $\geq 16$ & $\leq 1$ & 2 & $>2$ \\
\hline$C A Z$ & $\mathrm{MIC}(\mathrm{mg} / \mathrm{L})$ & $-\#$ & ${ }^{\#}$ & $\leq 2$ & $-\#$ & $-\#$ & -\# & $-\#$ &.$\#$ \\
\hline FEP & $\mathrm{MIC}(\mathrm{mg} / \mathrm{L})$ & $\leq 4$ & $>4$ & $-\#$ & $-\#$ & $-\#$ & $-\#$ & -\# & $-\#$ \\
\hline CIP & $\mathrm{MIC}(\mathrm{mg} / \mathrm{L})$ & $\leq 0.5$ & $>0.5$ & $\leq 1$ &.$\#$ & ${ }^{\#}$ & $\leq 0.5$ & - & $>0.5$ \\
\hline ERY & $\mathrm{MIC}(\mathrm{mg} / \mathrm{L})$ & $\leq 0.25$ & $>0.5$ & $\leq 0.5$ & $1-4$ & $\geq 8$ & $\leq 0.25$ & 0.5 & $>0.5$ \\
\hline MEM & $\mathrm{MIC}(\mathrm{mg} / \mathrm{L})$ & $\leq 2$ & $>2$ &.$^{\#}$ & $-\#$ & $-\#$ & $-^{\#}$ & $-{ }^{\#}$ & $-^{\#}$ \\
\hline TCY & D-zone(mm) & $\geq 28$ & $<25$ & $\geq 29$ & $25-28$ & $\leq 24$ & $\geq 22$ & - & $\leq 21$ \\
\hline $\mathrm{CHL}$ & D-zone(mm) & $\geq 30$ & $<30$ & -\# & $-\#$ & \# $^{-1}$ & -\# & -\# & -\# \\
\hline SXT & D-zone(mm) & $\geq 18$ & $<15$ & $\geq 13$ & $11-12$ & $\leq 10$ & $\geq 12$ & - & $\leq 11$ \\
\hline
\end{tabular}

${ }^{\mathrm{a}} M I C$ minimum inhibitory concentration, ${ }^{\mathrm{b}} D$-zone diameter of inhibition zone, ${ }^{\mathrm{c}}$ EUCAST, European Committee on Antimicrobial Susceptibility Testing, ${ }^{\mathrm{d}} C L S I$ Clinical and Laboratory Standards Institute, ${ }^{\mathrm{e}} B S A C$ British Society for Antimicrobial Chemotherapy, ${ }^{*} A M P$ ampicillin, $A M C$ amoxicillin-clavulanic acid, $C X M$ cefuroxime, CAZ ceftazidime, FEP cefepime, CIP ciprofloxacin, ERY eryciprofloxacin, MEM meropenem, TCY tetracycline, CHL chloramphenicol, SXT sulfamethoxazole-trimethoprim, \# no breakpoints listed in the according criterion

cases) and girls (7.7\%; 34/440 cases) in Zhongjiang County $\left(\chi^{2}=0.419, P=0.518\right)$, or in Youyang County $(9.0 \% ; 61 / 679$ cases and $10.7 \% ; 40 / 375$ cases, respectively, $\left.X^{2}=0.790, P=0.374\right)$.

Table 2 shows the susceptibility and the MICs of the isolates against 11 antibiotics. All isolates were susceptible to amoxicillin-clavulanic acid. The susceptibility rate to meropenem was $100 \%$ according to EUCAST; no equivalent breakpoints are listed in CLSI or BSAC. Regardless of the criteria used, the strains showed poor susceptibility to erythromycin. According to CLSI, the total non-susceptibility rate to erythromycin was $70.8 \%$ (Zhongjiang County, 79.2\%; Youyang County, 64.3\%), and this reached 92.1\% (Zhongjiang County, 90.9\%; Youyang County, 93.1\%) according to EUCAST or BSAC. The MICs of $21.9 \%$ (39/178 cases) of the isolates were $>256 \mathrm{mg} / \mathrm{L}$. Non-susceptibility to sulfamethoxazole-trimethoprim was significantly different between the two hospitals, regardless of the judgemnet criteria used, with isolates from Zhongjiang more susceptible than those from Youyang (Fisher's exact test, $P<0.05$ ). The total positive rate of $\beta$-lactamase was $99.4 \%$ (177/178 cases): $99.0 \%$ $(100 / 101$ cases) in Youyang County and 100\% (77/77 cases) in Zhongjiang County.

\section{Discussion}

Research into the antibiotic resistance of $M$. catarrhalis strains is limited, and the use of different judgemnet criteria makes it difficult to compare data between studies.Three criteria are commonly used in antimicrobial susceptibility testing for M. catarrhalis: EUCAST, CLSI, and BSAC. Clinical studies in China most commonly use CLSI. All isolates in the present study were susceptible to amoxicillin-clavulanic, ceftazidime, and ciprofloxacin according to CLSI, indicating that these antibiotics could be used for empirical treatment. The non-susceptibility rate to cefuroxime showed a significant difference according to different judgement criteria, being 13.5\% according to CLSI and EUCAST, but $96.0 \%$ according to BSAC. This is a source of confusion for clinicians. Previous reports documented high susceptibility rates of $M$. catarrhalis isolates to third- and fourth-generation cephalosporins (such as ceftriaxone and ceftazidime) and amoxicillinclavulanic, at nearly $100 \%$ [9, 11-14].

Isolates in the present study showed high resistance to erythromycin. Previous studies reported that 23S rRNA mutations A2330T and A2058T are major causes of resistance to macrolide antibiotics such as erythromycin [15-17]. Because the resistance of pathogens isolated from the respiratory tract to macrolide antibiotics is a serious concern in China, it is important to standardize the use of this antibiotic to help control and reduce the spread of resistance.

The $\beta$-lactamase positive rate in our study was high, at $99.4 \%$ (177/178 cases), which is in accordance with other recent findings $(96.5 \% \sim 100 \%)$ in China $[14,18,19]$. According to EUCAST, $M$. catarrhalis isolates producing $\beta$-lactamase should be reported as resistant to penicillins and aminopenicillins without inhibitors. However, we showed a resistance rate to ampicillin of $74.2 \%$ (Zhongjiang County, $81.8 \%$; Youyang County, 68.3\%) according to BSAC, suggesting that there is a discrepancy between $\beta$-lactamase production and resistance rates. We advise medical workers to pay attention to the high resistance rate and possible discrepancies in rates during their routine work. 


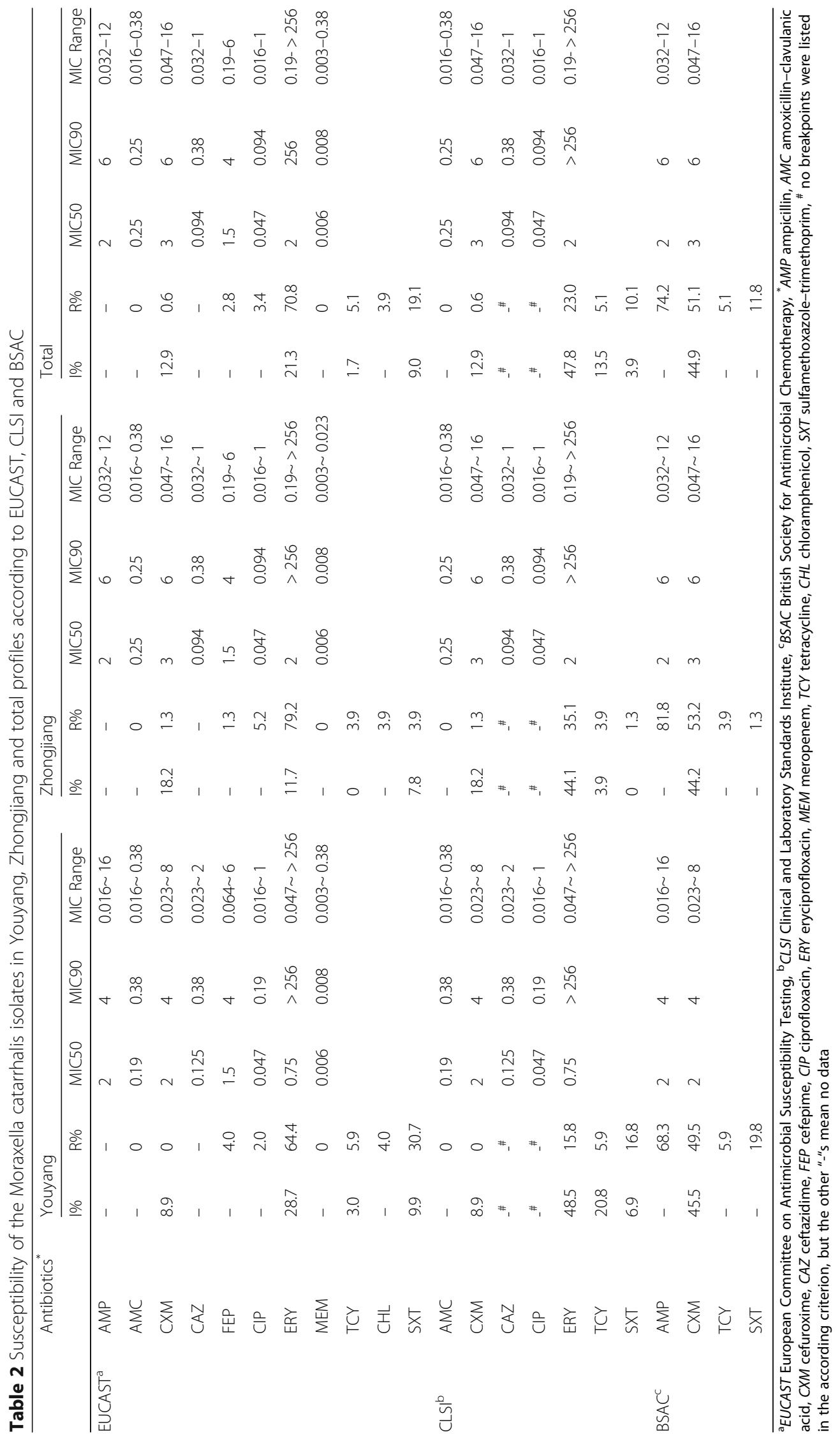




\section{Conclusions}

In summary, $99 \%$ of $M$. catarrhalis strains of the present study were shown to produce $\beta$-lactamase. The isolates showed high susceptibility to third- and fourth-generation cephalosporins and amoxicillin-clavulanic, and poor susceptibility to ampicillin and erythromycin. However, some notable discrepancies were observed between susceptibility rates obtained with different antibiotic resistance judgemnet criteria for $M$. catarrhalis isolates. Therefore, additional long-term surveys are required to monitor the antimicrobial resistance of this important human pathogen with the aim of standardizing criterion.

\section{Abbreviations \\ BSAC: British Society for Antimicrobial Chemotherapy; CAP: Community- acquired pneumonia; CLSI: Clinical and Laboratory Standards Institute; EUCAST: European Committee on Antimicrobial Susceptibility Testing; MIC: Minimum inhibitory concentration}

\section{Acknowledgments \\ We thank Sarah Williams, PhD, from Liwen Bianji, Edanz Group China (www.liwenbianji.cn), for editing the English text of a draft of this manuscript. Much thanks for the opportunity to show our research results from the 10th World Congress of the World Society for Pediatric Infectious Diseases (WSPID 2017) [20], and the 11th International Symposium on Antimicrobial Agents and Resistance and the 3rd International Interscience Conference on Infection and Chemotheropy (ISAAR \& ICIC 2017) [21]. \\ Funding \\ This study was financially supported by the Research Funds of Profession Quota Budget from the Beijing Municipal Science and Technology Commission (2016-bjsekyjs-3) and the medical research project of Chongqing Health and Family Planning Commission (No.2016ZDX041). The funding body had no role in the design of the study or collection, analysis, and interpretation of data or in writing the manuscript.}

\section{Availability of data and materials}

The data supporting the conclusion of this article are included within the article, and all of the datasets used and/or analyzed during the current study are available from the corresponding author.

\section{Authors' contributions}

WS, DW, CC, and KY designed the study. LY, WG, PT, and XC collected the isolates and performed the antimicrobial susceptibility test. WS, DW, CC, and KY collected the data, analyzed them, interpreted the results, and drafted the manuscript. All authors reviewed and revised the manuscript and approved the final version.

\section{Ethics approval and consent to participate}

A parent and/or legal guardian of each participant signed a written informed consent document before enrollment and before any study procedure was performed. This study was approved by the Ethics Committee of the two hospitals (Ethics Committee of People's Hospital of Zhongjiang County, and Ethics Committee of People's Hospital of Chongqing Youyang County). No ethical problems were encountered in this study.

\section{Consent for publication}

Not applicable

\section{Competing interests}

The authors declare that they have no competing interests.

\section{Publisher's Note}

Springer Nature remains neutral with regard to jurisdictional claims in published maps and institutional affiliations.

\section{Author details}

Key Laboratory of Major Diseases in Children, National Key Discipline of Pediatrics (Capital Medical University), National Clinical Research Center for Respiratory Diseases, Beijing Key Laboratory of Pediatric Respiratory Infection Diseases, Beijing Pediatric Research Institute, Beijing Children's Hospital, Capital Medical University, No. 56 Nan-li-shi Road, Beijing 100045, China. 2People's Hospital of Zhongjiang County, No. 96 Da-bei Street, Zhongjiang 618100, Sichuan, China. ${ }^{3}$ Youyang Hospital, the First Affiliated Hospital of Chongqing Medical University, People's Hospital of Chongqing Youyang County, No. 102 Tao-hua-yuan Road, Chongqing 409899, Youyang, China.

Received: 25 October 2017 Accepted: 28 June 2018

Published online: 20 July 2018

\section{References}

1. Aebi C. Moraxella catarrhalis-pathogen or commensal? Adv Exp Med Biol. 2011;697:107-16.

2. Ren D, Pichichero ME. Vaccine targets against Moraxella catarrhalis. Expert Opin Ther Targets. 2016;20(1):19-33.

3. Wang $H$, Chen $M, X u Y$, et al. Antimicrobial susceptibility of bacterial pathogens associated with community-acquired respiratory tract infections in Asia: report from the community-acquired respiratory tract infection pathogen surveillance (CARTIPS) STUDY, 2009-2010. Int J Antimicrob Agents. 2011:38(5):376-83.

4. Malmvall BE, Brorsson JE, Johnsson J. In vitro sensitivity to penicillin $V$ and beta-lactamase production of Branhamella catarrhalis. J Antimicrob Chemother. 1977;3(4):374-5.

5. Fung CP, Powell M, Seymour A, Yuan M, Williams JD. The antimicrobial susceptibility of Moraxella catarrhalis isolated in England and Scotland in 1991. J Antimicrob Chemother. 1992;30(1):47-55.

6. Doern GV, Jones RN, Pfaller MA, Kugler K. Haemophilus influenzae and Moraxella catarrhalis from patients with community-acquired respiratory tract infections: antimicrobial suscepitibility patterns from the SENTRY antimicrobial surveillance program (United States and Canada, 1997). Antimicrob Agents Chemother. 1999;43(2):385-9.

7. Pingault NM, Bowman JM, Lehmann D, Riley TV. Antimicrobial susceptibility of Moraxella catarrhalis isolated from children in Kalgoorlie-boulder, Western Australia. Pathology. 2010;42(3):273-9.

8. Clinical and Laboratory Standards Institute (CLSI). Methods for antimicrobial dilution and disk susceptibility testing of infrequently isolated or fastidious Bacteria; approved guideline. M45-A. 2014;26(19):28-9.

9. Andrews JM. BSAC working party on susceptibility testing. BSAC standardized disc susceptibility testing method (version 8). J Antimicrob Chemother. 2009;64(3):454-89.

10. Morrissey I, Maher K, Williams L, Shackcloth J, Felmingham D, Reynolds R et al. Non-susceptibility trends among Haemophilus influenzae and Moraxella catarrhalis from community -acquired respiratory tract infections in the UK and Ireland, 1999-2007 [J]. J Antimicrob Chemother, 2008, 62 Suppl 2:ii97-i103.

11. Bandet T, Whitehead S, Blondel-Hill E, Wagner K, Cheeptham N. Susceptibility of clinical Moraxella catarrhalis isolates in British Columbia to six empirically prescribed antibiotic agents. Can J Infect Dis Med Microbiol. 2014;25(3):155-8.

12. Abdullah FE, Ahuja KR, Kumar H. Prevalence and emerging resistance of Moraxella catarrhalis in lower respiratory tract infections in Karachi. J Pak Med Assoc. 2013;63(11):1342-4.

13. Che DD, Zhang H, Lu Q, Zhang YC, Lu M, Li WH. Study on Moraxella catarrhalis resistance and bro genotyping in children with acute lower respiratory tract infection. Chin J Pediatr. 2009;24(8):606-9. (in Chinese)

14. Luo YP. Analysis of pathogenic bacteria distribution and drug resistance in specimens of pediatric respiratory infection. J Modern Clin Med. 2016;42(1): 52-4. (in Chinese)

15. Liu Y, Xu H, Xu Z, Kudinha T, Fan X, Xiao M, Kong F, Sun H, Xu Y. High-level macrolide-resistant Moraxella catarrhalis and development of an allele-specific PCR assay for detection of 23S rRNA gene A2330 mutation: a three-year study at a Chinese tertiary hospital. Microb Drug Resist. 2015;21(5):507-11.

16. Iwata S, Sato Y, Toyonaga Y, Hanaki H, Sunakawa K. Genetic analysis of a pediatric clinical isolate of Moraxella catarrhalis with resistance to macrolides andquinolones. J Infect Chemother. 2015;21(4):308-11.

17. Kasai A, Ogihara S, Yamada K, Tanimichi Y, Nishiyama H, Saito R. Prevalence and olecular analysis of macrolide-resistant Moraxella catarrhalis clinical 
isolates in Japan, following emergence of the highly macrolide-resistant strain NSH1 in 2011. J Med Microbiol. 2015;64(7):708-13.

18. Wang PJ, Xie CB, Wu YL. Antimicrobial resistance and BRO genotyping of Moraxella catarrhalis isolates from respiratory tract in children. J Clin Pediatr. 2013;31(8):719-22. (in Chinese)

19. Lin AX, Tan N, Yang XH, Li LL. The specimen distribution and antibiotic susceptibility analysis of 997 Moraxella catarrhalis isolates. Int I Lab Med. 2016;37(3):376-7. (in Chinese)

20. Yao K, Shi W. $\beta$-Lactamase production and antibiotic susceptibility pattern of Moraxella catarrhalis isolates collected from two county hospitals in China. WSPID 2017; WSPD7-0415. https://wspid2017.kenes.com/Documents/ WSPID17_-all\%20abstracts.pdf. Accessed 2 Dec 2017.

21. Shi W, Yao K. $\beta$-Lactamase production and antibiotic susceptibility pattern of Moraxella catarrhalis isolates collected from two county hospitals in China. ISAAR\& ICIC 2017; P1-GN03. https://www.sciencedirect.com/science/ article/pii/S0924857917303424?via\%3Dihub. Int J Antimicrob Agents 50 S1 (2017) S94.

Ready to submit your research? Choose BMC and benefit from:

- fast, convenient online submission

- thorough peer review by experienced researchers in your field

- rapid publication on acceptance

- support for research data, including large and complex data types

- gold Open Access which fosters wider collaboration and increased citations

- maximum visibility for your research: over $100 \mathrm{M}$ website views per year

At BMC, research is always in progress.

Learn more biomedcentral.com/submissions 